\title{
Health care utilization and overall costs based on opioid dependence in patients undergoing surgery for degenerative spondylolisthesis
}

\author{
Mayur Sharma, MD, MCh, Beatrice Ugiliweneza, PhD, MSPH, Zaid Aljuboori, MD, and \\ Maxwell Boakye, MD, MPH, MBA \\ Department of Neurosurgery, University of Louisville, Kentucky
}

\begin{abstract}
OBJECTIVE Opioid abuse is highly prevalent in patients with back pain. The aim of this study was to identify health care utilization and overall costs associated with opioid dependence in patients undergoing surgery for degenerative spondylolisthesis (DS).

METHODS The authors queried the MarketScan database using ICD-9 and CPT-4 codes from 2000 to 2012. Opioid dependency was defined as having a diagnosis of opioid use disorder, having a prescription for opioid use disorder, or having 10 or more opioid prescriptions. Opioid dependency was evaluated in 12-month period leading to surgery and in the period 3-15 months following the procedure. Patients were segregated into 4 groups based on opioid dependence before and after surgery: group NDND (prior nondependent who remain nondependent), group NDD (prior nondependent who become dependent), group DND (prior dependent who become nondependent), and group DD (prior dependent who remain dependent). The outcomes of interest were discharge disposition, hospital length of stay (LOS), complications, and health care resource costs. The 4 groups were compared using the Kruskal-Wallis test and linear contrasts built from generalized regression models.
\end{abstract}

RESULTS A total of 10,708 patients were identified, with $81.57 \%, 3.58 \%, 8.54 \%$, and $6.32 \%$ of patients in groups NDND, NDD, DND, and DD, respectively. In group DD, $96.31 \%$ of patients had decompression with fusion, compared with $93.59 \%$ in group NDND. Patients in group NDD, DND, and DD had longer hospital LOS compared with those in group NDND. Patients in group DD were less likely to be discharged home compared with those in group NDND (odds ratio $0.639,95 \%$ confidence interval $0.52-0.785$ ). At $3-15$ months postdischarge, patients in group DD incurred $21 \%$ higher hospital readmission costs compared with those in group NDND. However, patients in groups NDD and DD were likely to incur 2.8 times the overall costs compared with patients in group NDND $(p<0.001)$ at $3-15$ months after surgery (median overall payments: group NDD $\$ 20,033$ and group DD $\$ 19,654$, vs group NDND $\$ 7994$ ).

CONCLUSIONS Patients who continued to be opioid dependent or became opioid dependent following surgery for DS incurred significantly higher health care utilization and costs within 3 months and in the period 3-15 months after discharge from surgery.

https://thejns.org/doi/abs/10.3171/2018.2.FOCUS17764

KEYWORDS overall costs; health care utilization; fusion; decompression; opioid dependence; degenerative spondylolisthesis

$\mathrm{N}$ ARCOTICS use in the US has significantly increased in the past two decades, resulting in a national opioid crisis. ${ }^{16}$ The Centers for Disease Control and Prevention reported that $73 \%$ of deaths from prescription drug overdoses are caused by opioid overdose. ${ }^{26}$ Opioid use is highly prevalent in patients with back pain either prior to or following surgery. ${ }^{1,3,5,22}$ Degenerative spondylolisthesis (DS) is a common condition with a prevalence of approximately $6 \%$ in the US, affecting elderly patients with a female predilection. ${ }^{15}$ A majority of these patients

ABBREVIATIONS $\mathrm{Cl}=$ confidence interval; $\mathrm{DD}=$ prior dependent who remains dependent; $\mathrm{DND}=$ prior dependent who becomes nondependent; $\mathrm{DS}=$ degenerative spondylolisthesis; DVT = deep vein thrombosis; ED = emergency department; ER = emergency room; IQR = interquartile range; LOS = length of stay; NDD = prior nondependent who becomes dependent; NDND = prior nondependent who remains nondependent; NIS = Nationwide Inpatient Sample; OR = odds ratio; PE = pulmonary embolism; $\mathrm{RR}$ = relative risk

SUBMITTED December 7, 2017. ACCEPTED February 6, 2018.

INCLUDE WHEN CITING DOI: 10.3171/2018.2.FOCUS17764. 
are being managed with decompression and fusion in the US, with perioperative opioid use. . $^{4,17,21,23,28}$ Several studies have evaluated the relationship between spine surgery and long-term opioid use , $^{8,28}, 30$ Approximately $0.1 \%$ of patients with underlying mental health disorders have been shown to continue prolonged opioid use following surgery., $4,821,28$

Opioid dependency in these patients causes significant financial burden on the health care system and also affects quality of life. . $^{12,14,31}$ The total economic burden of opioid overdose, abuse, and dependency has been estimated to be $\$ 78.5$ billion in the US..$^{12,24}$ The mean adjusted peruser total cost of prescription medications for spine problems increased from \$166 to \$397 (139\%) over the past decade. Increasing use of preoperative opioids has been shown to be associated with prolonged hospital length of stay (LOS; each 100-mg morphine equivalent extends the hospital LOS by 1.1 days), delay in return to work, adverse functional outcome, and postoperative opioid dependency. ${ }^{1,3-5,8,22}$

Recently, there has been widespread use of national databases to answer clinical questions on a larger scale. ${ }^{2,8,9,21,29}$ To that end, we aimed to identify health care utilization and overall costs based on opioid dependence in patients undergoing surgery for DS using a national database. To the best of our knowledge, this is the first study focusing on the economic burden related to the surgical management of this patient cohort.

\section{Methods \\ Data Source}

We used MarketScan data from Truven Health Analytics, part of IBM Watson Health. This database is constructed using paid insurance claims from approximately 100 payers and is available to researchers for a fee. Included in MarketScan are longitudinal clinical utilization of health care resources, insurance enrollment, costs associated with the treatment, and provider information ${ }^{13}$ across different services over the time of patient enrollment for covered individuals and their dependents. The data contain a unique encrypted identification number for each patient that is used to extract data across different files. ${ }^{11}$ It has been used in medical and outcomes research since $1990 .{ }^{13}$ We used a custom file consisting of neurology/neurosurgery inpatient, outpatient, and prescription medication data files from commercial claims and encounters, and Medicare supplemental and Medicaid databases. For this study, we extracted data related to health care utilization and costs associated with opioid dependence in patients undergoing surgery for DS.

\section{Patient Selection, Exploratory Variables, and Follow-Up}

We used the inpatient admission tables to extract patients with a primary diagnosis of acquired lumbar spondylolisthesis (ICD-9-CM code 738.4) with a concurrent procedure of either a laminectomy or fusion using ICD9 and CPT- 4 codes (laminectomy: ICD-9 codes 03.0 or 03.09, or CPT-4 codes 63005, 63012, 63017, 63030, 63042, 63047, 63056, and 63102; fusion: ICD-9 codes 81.06, 81.07, and 81.08 , or CPT- 4 codes 22558,22630 , and 22612). Cases with concurrent refusion (ICD-9 codes 81.36, 81.37, and
81.38; CPT-4 codes 22830, 22849, 22850, 22852, 22855) or under 18 years of age were excluded. For each patient, the first occurring case satisfying the above conditions was flagged as the index hospitalization.

Patient characteristics at the index hospitalization including age, sex, year of index hospitalization, insurance type (commercial, Medicaid, Medicare), comorbidities, and instrumentation and number of levels were used as the variables. We used the Elixhauser comorbidity score,$^{10}$ which was calculated based on modified ICD-9-CM codes (Quan et al. ${ }^{27}$ ). Cases were classified as instrumented if the CPT-4 procedure codes 22840-22847 were present in the claims. Similarly, cases were classified as multiple-level surgery if ICD-9 procedure codes $81.62,81.63$, 81.64 , or CPT- 4 procedure codes $22585,22632,22614$, $63035,63044,63048,63057,63103$, or 22842-22847 were present.

To calculate the look-back period (preoperative follow-up time) and the follow-up time, we used the dates of start and end enrollment times according to the formulas below:

Pre-follow-up time $=$ surgery admission date - start enrollment date

Post-follow-up time $=$ end enrollment date - surgery discharge date

If the start or end enrollment dates fell outside our data, they were censored, respectively, with the beginning of our data (January 1,2000 ) or the last date of our data (December 31,2012).

\section{Opioid Dependence and Analysis Groups}

Presurgery (within 12 months prior to the procedure) and postsurgery opioid dependency (3-15 months following discharge) was determined using either ICD-9 codes for opioid use $(305.50,305.55,305.52,304.00,304.01$, $304.02,304.70,304.71$ or 304.72$) ;{ }^{7}$ at least 10 prescriptions of opioid medications ${ }^{8,20}$ such as buprenorphine, butorphanol, codeine phosphate, dihydrocodeine, fentanyl citrate, hydrocodone, hydromorphone, levorphanol, meperidine, methadone, morphine sulfate, nalbuphine, opium alkaloids, oxycodone, oxymorphone, pentazocine, propoxyphene, tapentadol, tramadol, buprenorphine, or naltrexone $;{ }^{19}$ or a prescription for medications (buprenorphine or naltrexone)/psychotherapy (CPT-4 code $4306 \mathrm{~F})^{7}$ related to the management of opioid addiction disorder during the study period.

We defined the comparative groups based on opioid dependence before and after surgery as follows: group NDND (prior nondependent who remains nondependent), group NDD (prior nondependent who becomes dependent), group DND (prior dependent who becomes nondependent), and group DD (prior dependent who remains dependent).

\section{Postoperative Outcomes}

The outcomes of interest in this study were: LOS during index hospitalization following surgery; discharge disposition; complications (renal, cardiac, general neurosurgi- 
TABLE 1. ICD-9-CM codes used for extracting complications from the database

\begin{tabular}{cc}
\hline Complication Type & ICD-9-CM Codes \\
\hline Renal & $584,584.5-584.9 ; 997.5$ \\
\hline Cardiac & $997.1 ; 410.0 ; 410.00 ; 410.01 ; 410.1 ; 410.10 ; 410.11 ; 410.2 ; 410.20 ; 410.21 ; 410.3 ; 410.30 ; 410.31 ; 410.4 ; 410.40 ; 410.41 ; 410.5 ;$ \\
& $410.50 ; 410.51 ; 410.6 ; 410.60 ; 410.61 ; 410.7 ; 410.70 ; 410.71 ; 410.8 ; 410.80 ; 410.81 ; 410.9 ; 410.90 ; 410.91$ \\
\hline Neural & $997.00-997.09$ \\
\hline DVT \& PE & $415.1 ; 415.11 ; 415.19 ; 451.11 ; 415.11 ; 451.19 ; 451.12 ; 451.81 ; 451.9453 .4 ; 453.40 ; 453.41 ; 453.42 ; 453.8 ; 453.9$ \\
\hline Pulmonary & $507.3 ; 518.4 ; 518.5 ; 518.81 ; 518.82 ; 997.3 ; 997.31 ; 997.39$ \\
\hline Infection & $510,510.0,510.9 ; 038,038.0-038.9,038.10-038.19,038.40-038.49 ; 320,320.0-320.9,320.81-320.89 ; 513,513.0,513.1 ;$ \\
& $519.2 ; 590.1,590.10,590.11 ; 590.80 ; 683$ \\
\hline Wound & $998.1-998.9,998.11-998.13,998.30-998.33,998.51,998.59,998.81-998.89$ \\
\hline
\end{tabular}

cal, general neurological, deep vein thrombosis [DVT] or pulmonary embolism [PE], pulmonary, infection, wound infection, hydrocephalus, mechanical ventilation, laceration, or CSF rhinorrhea); health care resource use (hospital days, 30-day hospital readmission, and emergency department [ED]) and health care resource costs; outpatient services; and medication refills, with their associated payments among the 4 groups (NDND, NDD, DND, DD).

\section{Complications}

The ICD-9 codes used to search for complications are listed in Table 1. The incidence of complications was evaluated during the index hospitalization and 30 days after surgery. The presence of a complication was noted as the occurrence of any of the complication types described above. Index hospitalization complications were complications that occurred during the index procedure hospitalization. Thirty-day complications were evaluated by examining all hospitalizations and/or outpatient visits within 30 days after surgery discharge.

\section{Health Care Resources Use}

We investigated the index hospitalization and the postdischarge health care resources use. For the index hospitalization, we investigated the LOS and discharge disposition. For postdischarge health resource use outcomes, we focused on 30-day hospital readmission and outpatient emergency room (ER) use, 3-month and 3-15-month hospital admissions, outpatient services, and medication refills.

\section{Health Care Resources Payment}

We evaluated all the payments associated with the uses described in the paragraph above: index hospitalization payment, and postdischarge inpatient, outpatient, and medication payments. Payments were cumulated over all hospitalizations (inpatient payment), all outpatient services (outpatient payments), all prescription medication refills (medications payments), and a combination of all three. Outpatient ER services were a subset of the outpatient services with an indicator that they occurred in the ED. All payments were inflated to 2012 US dollars using the medical component of the consumer price index, which can be accessed through the website of the US Bureau of Labor Statistics. $^{25}$

\section{Statistical Analysis}

We summarized continuous variables using means and standard deviations, medians and interquartile ranges (IQRs), as well as the full range (minimum to maximum). Categorical variables were summarized using counts and percentages. To compare the patient characteristics and outcomes among different opioid use groups, we used the Kruskal-Wallis test for continuous variables and the chisquare test for categorical variables. ${ }^{18}$ We also performed a multivariable analysis for each outcome using the linear regression on log-transformed values for continuous outcomes and the logistic regression for categorical outcomes. The covariate procedure types, sex, age, Elixhauser index, insurance type, and number of levels were added to the model in addition to the opioid dependence group. From these models, we build linear contrasts to compare different groups to the reference group (NDND). Adjusted comparisons were presented in terms of relative risk (RR) for continuous variables and odds ratio (OR) for categorical variables. We used SAS software (version 9.4, SAS Institute, Inc.) for data preprocessing and data analysis. $^{32}$

\section{Results}

\section{Patient Demographics}

A cohort of 10,708 patients who underwent surgery for DS was identified using the MarketScan database: $81.57 \%$, $3.58 \%, 8.54 \%$, and $6.32 \%$ of patients were in groups NDND, NDD, DND, and DD, respectively. The overall median age was 61 years and $65.1 \%$ of patients were females. Group DD had significantly more younger females compared to the NDND group (median age 57 vs 61 years, female sex $69.7 \%$ vs $64.2 \%$ ). Similarly, significantly more patients in group DD had primarily commercial insurance (69.4\%) compared with those in groups NDND (60.7\%), NDD $(64.5 \%)$, and DND (60.5\%; $\mathrm{p}<0.001)$. There was no significant difference in the Elixhauser comorbidity index among the different groups $(\mathrm{p}=0.3493)$. Overall, the majority of patients $(94 \%)$ underwent decompression with fusion for DS: $96.3 \%$ of patients in group DD had decompression with fusion compared with $93.6 \%$ in group NDND, however, the majority of patients who underwent decompression alone were in group NDND compared to those in group DD (6.41\% vs $3.69 \%)$. The demographics 
TABLE 2. Demographics stratified by opioid dependency

\begin{tabular}{|c|c|c|c|c|c|c|}
\hline \multirow[b]{2}{*}{ Variable } & \multicolumn{6}{|c|}{ Opioid Dependency Before \& After Surgery } \\
\hline & NDND & NDD & DND & DD & $p$ Value & Combined Cohort \\
\hline No. of patients (\%) & $8734(81.57)$ & $383(3.58)$ & $914(8.54)$ & $677(6.32)$ & & $10,708(100)$ \\
\hline \multicolumn{7}{|l|}{ Demographics } \\
\hline Age, yrs & & & & & $<0.0001$ & \\
\hline Mean (SD) & $61.16(11.85)$ & $58.02(13.17)$ & $60.27(11.54)$ & $56.39(11.37)$ & & $60.67(11.91)$ \\
\hline Median (IQR) & $61(54-70)$ & $58(49-67)$ & $60(53-69)$ & $57(49-63)$ & & $61(54-69)$ \\
\hline Range & 18-95 & 22-92 & 19-86 & $20-86$ & & 18-95 \\
\hline Females, no. (\%) & $5612(64.25)$ & $259(67.62)$ & $632(69.15)$ & $472(69.72)$ & 0.0008 & $6975(65.14)$ \\
\hline Insurance, no. (\%) & & & & & $<0.0001$ & \\
\hline Commercial & $5301(60.69)$ & $247(64.49)$ & $553(60.5)$ & $470(69.42)$ & & $6571(61.37)$ \\
\hline Medicaid & $275(3.15)$ & $23(6.01)$ & $47(5.14)$ & $46(6.79)$ & & $391(3.65)$ \\
\hline Medicare & $3158(36.16)$ & $113(29.5)$ & $314(34.35)$ & $161(23.78)$ & & $3746(34.98)$ \\
\hline Elixhauser index, no. (\%) & & & & & 0.3493 & \\
\hline 0 & $4024(46.07)$ & $187(48.83)$ & $417(45.62)$ & $326(48.15)$ & & $4954(46.26)$ \\
\hline 1 & $2777(31.8)$ & $111(28.98)$ & $287(31.4)$ & $211(31.17)$ & & $3386(31.62)$ \\
\hline 2 & $1247(14.28)$ & $53(13.84)$ & $129(14.11)$ & $75(11.08)$ & & $1504(14.05)$ \\
\hline $3+$ & $686(7.85)$ & $32(8.36)$ & $81(8.86)$ & $65(9.6)$ & & $864(8.07)$ \\
\hline Surgery type, no. (\%) & & & & & 0.0009 & \\
\hline Decompression w/o fusion & $560(6.41)$ & $18(4.70)$ & $37(4.05)$ & $25(3.69)$ & & $640(5.97)$ \\
\hline Decompression w/ fusion & $8174(93.59)$ & $365(95.3)$ & $877(95.95)$ & $652(96.31)$ & & 10,068 (94.02) \\
\hline Multiple levels & $6627(75.88)$ & $304(79.37)$ & $685(74.95)$ & $507(74.89)$ & 0.3409 & $8123(75.86)$ \\
\hline
\end{tabular}

Boldface type indicates statistical significance.

and other patient and treatment characteristics are noted in Table 2.

\section{Index Hospitalization Outcomes}

Overall, the median hospital LOS was 3 days, with the longest in the NDD group (4 days; Table 3 ). Compared to the NDND group, the hospital LOS was significantly longer $(\mathrm{p}<0.001)$ in groups NDD (RR 1.058, 95\% CI 1.005-1.114), DND (RR 1.087, 95\% CI 1.05-1.124), and DD (RR 1.088, 95\% CI 1.046-1.132; Table 4).

In all, 83.55\% of patients $(\mathrm{n}=8947)$ were discharged home and 9.5\% $(\mathrm{n}=1018)$ had in-hospital complications. Patients in groups DD and DND were less likely to be discharged home compared with those in group NDND (OR $0.639,95 \%$ CI $0.52-0.785$, and OR $0.828,95 \%$ CI $0.691-$ 0.992). No significant difference in terms of in-hospital complications was noted among the groups $(p=0.9062)$.

The median cost of index hospitalization for the combined cohort was $\$ 40,095$. Patients in the DD group had a median cost of $\$ 43,524$, compared with $\$ 39,654$ for patients in group NDND, $\$ 41,052$ in group NDD, and $\$ 41,027$ in group DND ( $p=0.0351)$. However, on adjusted analysis, these differences were not significant $(\mathrm{p}=0.7814$; Tables 3 and 4, Fig. 1).

\section{Thirty-Day Postdischarge Outcomes}

At 30 days after discharge, $6.79 \%(\mathrm{n}=727), 3.46 \%$ (n $=371)$, and $6.47 \%(n=693)$ of all patients were noted to have complications, hospital readmissions, and ER readmissions, respectively. However, there was no significant difference among the groups in terms of complications $(\mathrm{p}>0.05)$.

Patients in the NDD group had the highest number of patients with hospital (7.05\%) and ER $(9.4 \%)$ readmissions at 30 days follow-up compared with those in other groups. On adjusted analysis, patients in the NDD group were 2.1 times and 61\% more likely to have hospital and ER readmissions compared with those in the NDND group $(\mathrm{p}=$ 0.023 and 0.008 , respectively; Tables 3 and 4).

\section{Postdischarge Outcomes at 3 Months and 3-15 Months}

At 3 months following discharge, $6.12 \%(n=655)$ of all patients were readmitted. Compared to patients in the NDND group, patients in the NDD, DND, and DD groups were 2.2 times, $42 \%$, and $88 \%$ more likely to have hospital readmissions $(\mathrm{p}<0.001)$. Patients in the DD group incurred $42 \%$ higher outpatient costs, 2.6 times higher medication refill costs, and 2.1 times higher overall costs compared with those in the NDND group ( $p<0.001$, Fig. 2).

At 3-15 months after discharge, overall $19 \%$ of patients were readmitted, and median outpatient cost, medication cost, and overall costs were $\$ 4831$, $\$ 1959$, and $\$ 8987$, respectively. Patients who were opioid dependent after surgery (groups NDD and DD) were 3.6 and 2.9 times more likely to have hospital readmissions compared to those in the NDND group. Also, patients in group DD incurred $21 \%$ higher hospital readmission costs compared to those in the NDND group. Patients in groups NDD and DD were likely to incur 2.8 times the overall costs compared to patients in group NDND $(\mathrm{p}<0.001)$ at $3-15$ months 


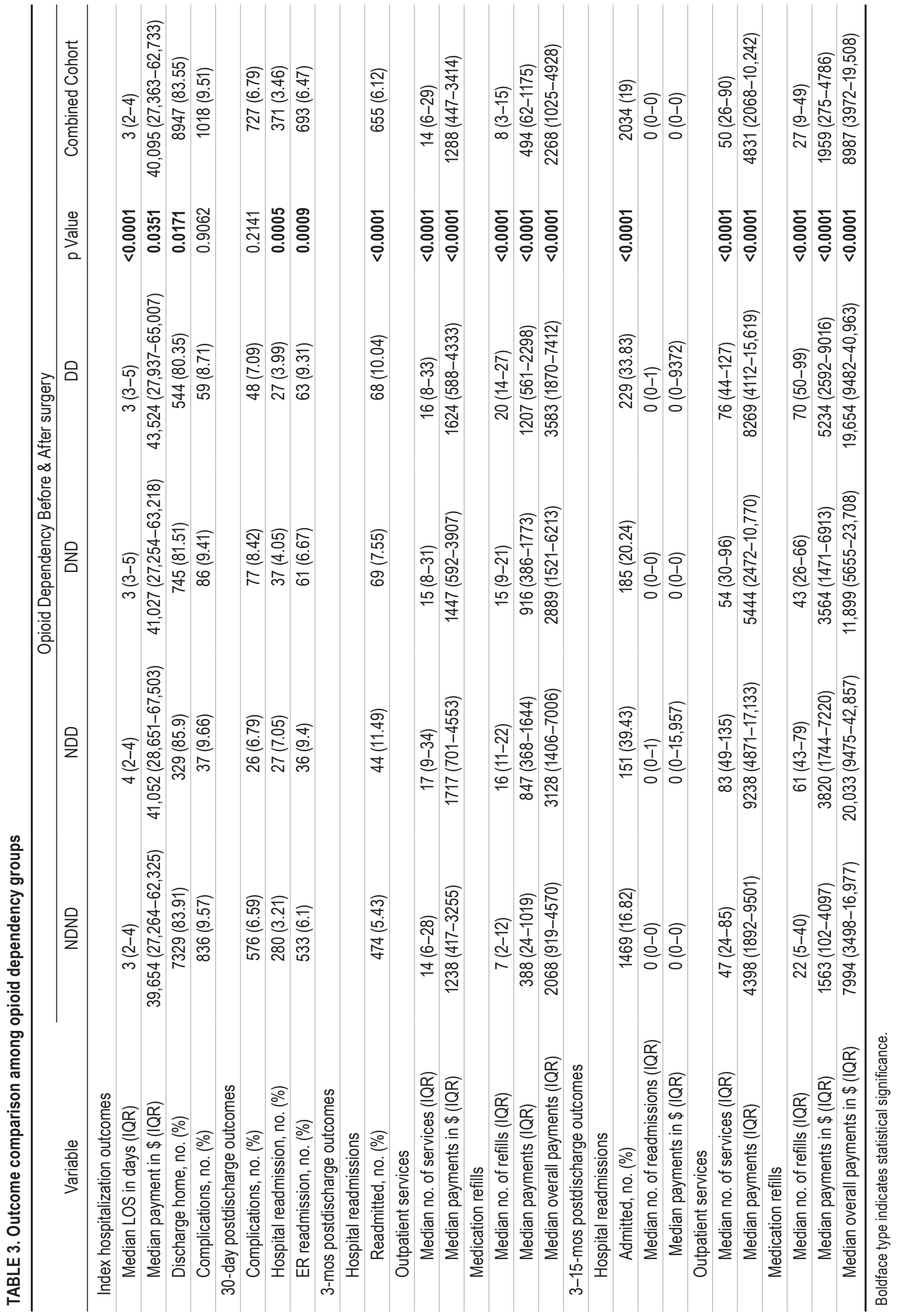


TABLE 4. Outcome-adjusted comparison among opioid dependency groups

\begin{tabular}{|c|c|c|c|c|c|}
\hline \multirow[b]{2}{*}{ Variable } & \multicolumn{5}{|c|}{ Opioid Dependency Before \& After Surgery $(95 \% \mathrm{Cl})$} \\
\hline & NDND & NDD & DND & DD & $\mathrm{p}$ Value \\
\hline \multicolumn{6}{|l|}{ Index hospitalization outcomes } \\
\hline LOS, RR & Ref & $1.058(1.005-1.114)$ & $1.087(1.05-1.124)$ & $1.088(1.046-1.132)$ & $<0.0001$ \\
\hline Payment, RR & Ref & $1.036(0.938-1.144)$ & $1.021(0.955-1.091)$ & $1.024(0.949-1.106)$ & 0.7814 \\
\hline Discharge home, OR & Ref & $1.074(0.795-1.452)$ & $0.828(0.691-0.992)$ & $0.639(0.52-0.785)$ & $<0.0001$ \\
\hline Complications, OR & Ref & $1.057(0.744-1.501)$ & $0.992(0.784-1.256)$ & $1.007(0.76-1.333)$ & 0.9914 \\
\hline \multicolumn{6}{|l|}{ 30-day postdischarge outcomes } \\
\hline Complications, OR & Ref & $1.085(0.72-1.634)$ & $1.318(1.026-1.692)$ & $1.201(0.881-1.636)$ & 0.1322 \\
\hline Hospital readmission, OR & Ref & $2.175(1.44-3.286)$ & $1.256(0.884-1.786)$ & $1.134(0.755-1.703)$ & 0.0023 \\
\hline ER readmission, OR & Ref & $1.615(1.13-2.308)$ & $1.08(0.82-1.423)$ & $1.613(1.221-2.131)$ & 0.0008 \\
\hline \multicolumn{6}{|l|}{ 3-mos postdischarge outcomes } \\
\hline \multicolumn{6}{|l|}{ Hospital admissions } \\
\hline Admitted, OR & Ref & $2.258(1.622-3.142)$ & $1.426(1.095-1.856)$ & $1.887(1.439-2.475)$ & $<0.0001$ \\
\hline \multicolumn{6}{|l|}{ Outpatient services } \\
\hline No. of services, RR & Ref & $1.217(1.192-1.242)$ & $1.07(1.054-1.085)$ & $1.204(1.185-1.223)$ & $<0.0001$ \\
\hline Payments, RR & Ref & $1.463(1.259-1.699)$ & $1.225(1.108-1.353)$ & $1.428(1.272-1.603)$ & $<0.0001$ \\
\hline \multicolumn{6}{|l|}{ Medication refills } \\
\hline No. of refills, RR & Ref & $2.086(2.034-2.139)$ & $1.894(1.861-1.928)$ & $2.66(2.613-2.708)$ & $<0.0001$ \\
\hline Payments, RR & Ref & $1.806(1.578-2.067)$ & $1.743(1.593-1.907)$ & $2.607(2.352-2.89)$ & $<0.0001$ \\
\hline Median overall payments, RR & Ref & $1.809(1.575-2.077)$ & $1.562(1.425-1.713)$ & $2.118(1.904-2.356)$ & $<0.0001$ \\
\hline \multicolumn{6}{|l|}{ 3-15 mos postdischarge outcomes } \\
\hline \multicolumn{6}{|l|}{ Hospital admissions } \\
\hline Readmitted, OR & Ref & $3.592(2.888-4.468)$ & $1.279(1.075-1.521)$ & $2.936(2.467-3.495)$ & $<0.0001$ \\
\hline No. of readmissions, $\mathrm{RR}$ & Ref & $2.603(2.255-3.005)$ & $1.203(1.051-1.376)$ & $2.5(2.223-2.811)$ & $<0.0001$ \\
\hline Payments, RR & Ref & $1.156(0.961-1.391)$ & $0.875(0.739-1.036)$ & $1.217(1.041-1.422)$ & 0.0085 \\
\hline \multicolumn{6}{|l|}{ Outpatient services } \\
\hline No. of services, RR & Ref & $1.544(1.528-1.56)$ & $1.131(1.122-1.14)$ & 1.48 (1.468-1.492) & $<0.0001$ \\
\hline Payments, RR & Ref & $2.149(1.873-2.466)$ & $1.245(1.136-1.365)$ & $2.01(1.808-2.235)$ & $<0.0001$ \\
\hline \multicolumn{6}{|l|}{ Medication refills } \\
\hline No. of refills, RR & Ref & $2.471(2.439-2.503)$ & $1.826(1.807-1.844)$ & $2.969(2.941-2.998)$ & $<0.0001$ \\
\hline Payments, RR & Ref & $2.291(1.985-2.643)$ & $1.78(1.616-1.961)$ & $3.132(2.805-3.498)$ & $<0.0001$ \\
\hline Overall payments, RR & Ref & $2.778(2.436-3.169)$ & $1.509(1.382-1.647)$ & $2.853(2.578-3.156)$ & $<0.0001$ \\
\hline
\end{tabular}

Adjusted comparisons were obtained from linear contrasts derived from multivariable regression models that included the covariates of procedure type, sex, age,

Elixhauser index, insurance, and number of levels, in addition to opioid dependency group. Boldface type indicates statistical significance.

after surgery (median overall costs: group NDD $\$ 20,033$ and group DD \$19,654, vs group NDND \$7994; Tables 3 and 4, Figs. 3 and 4).

\section{Discussion}

In our cohort of 10,708 patients, we found that patients who remain opioid dependent (DD and NDD) after surgery for DS had longer hospital LOSs and were less likely to be discharged home compared with those who were opioid nondependent prior to surgery and remained nondependent (NDND) after surgery. Also, at 3-15 months, patients in the DD group incurred $21 \%$ higher hospital readmission costs compared with those in the NDND group. Patients in groups NDD and DD were likely to incur 2.8 times the overall costs compared with patients in group NDND, causing a significant health care burden.
The incidence of opioid dependence following major surgery in opioid-naïve patients varies from $0.1 \%$ to $0.4 \%, 4,8,21,28,31$ Open procedures, preprocedure opioid dependence, and mental illness are identified as risk factors for developing long-term opioid dependence. ${ }^{4,8,28,31}$ In our study, we found that $3.58 \%$ of opioid-naïve patients developed new-onset opioid dependence following surgery for DS.

Using the Nationwide Inpatient Sample (NIS), a recent study reported that 48,911 patients underwent surgery for DS in the US from 2001 to $2010^{23}$ with a substantial increase in the use of interbody fusion techniques in the last decade. ${ }^{17,23}$ In this study, $64 \%$ of patients were female and the average age was 59.7 years, ${ }^{23}$ which are demographics comparable to our study (65\% female and mean age 61 years). The majority of patients in this study underwent posterior/transforaminal lumbar interbody fusion-only 

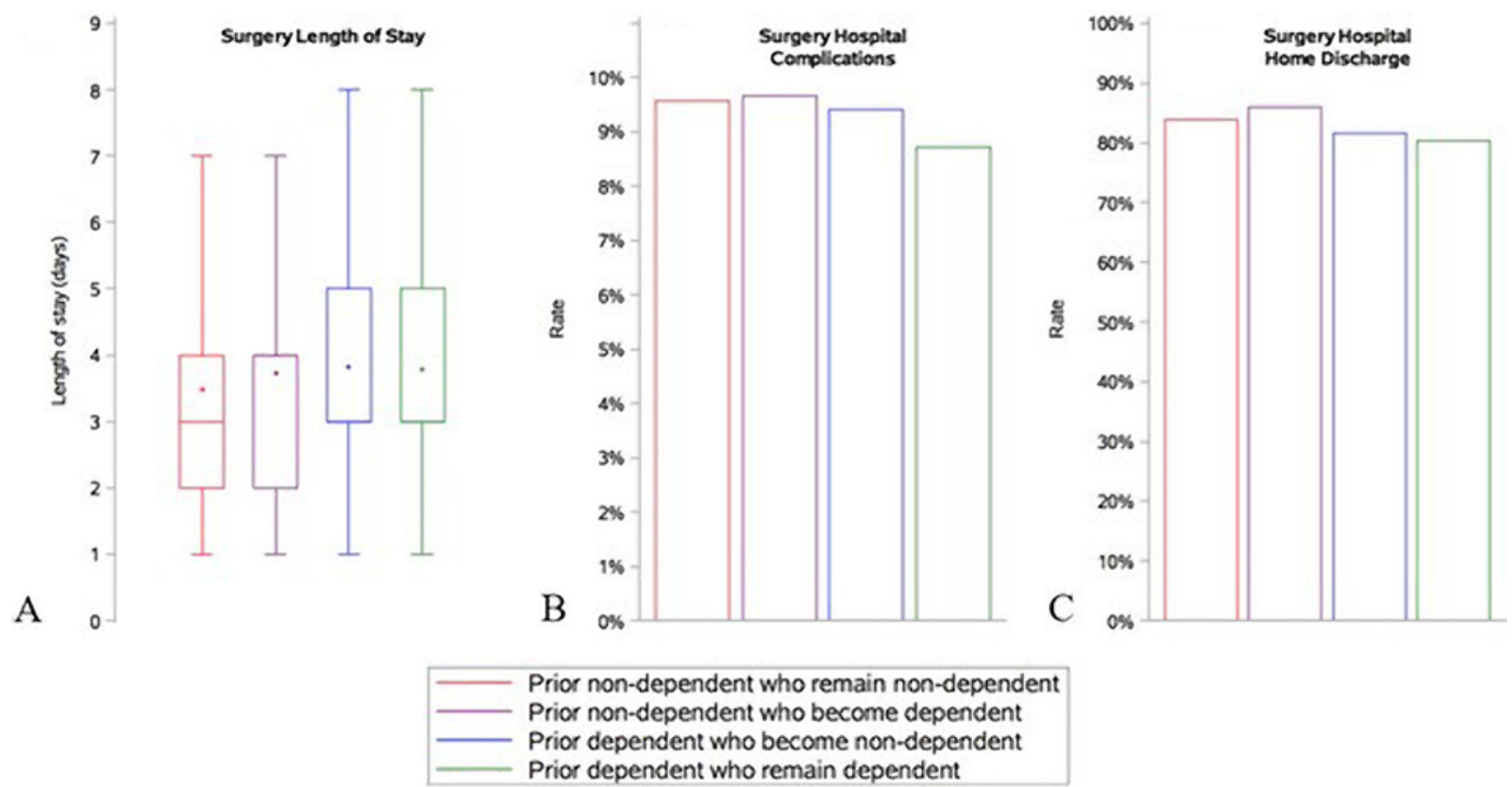

FIG. 1. Bar graphs showing the distribution of hospital LOS (A), in-hospital complication rates (B), and hospital home discharge rates $(C)$ among patients in the NDND, NDD, DND, and DD groups following surgery for DS.

procedures $(83.9 \%, \mathrm{n}=41,051)$ followed by posterolateral fusion-only procedures $(10.2 \%, \mathrm{n}=4989)$ for DS in this study. ${ }^{23}$

In our study, the median LOS for the combined cohort was 3 days (range 2-4 days). Our results are comparable to another NIS-based study, ${ }^{23}$ which reported an average LOS of 4.4 days. We found that, compared to the NDND group, the median LOS was significantly longer in the NDD, DND, and DD groups ( $\mathrm{p}<0.001)$. In contrast, Walid et al. ${ }^{33}$ reported that there was no correlation $(\mathrm{p}>$ 0.1 ) between preoperative opioid dependence and LOS in a cohort of 150 patients who underwent surgery for a variety
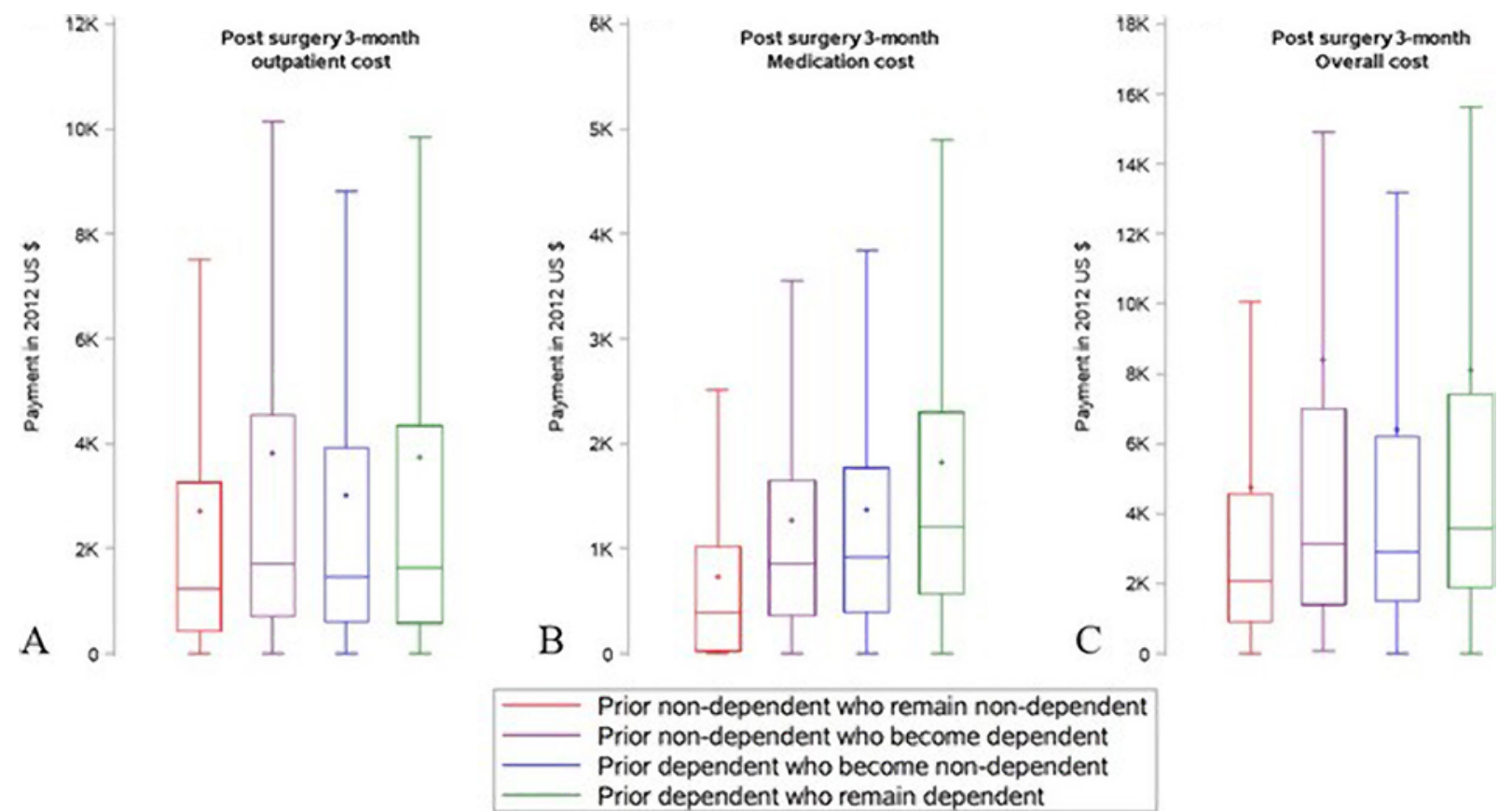

FIG. 2. Bar graphs showing the median outpatient costs (A), medication costs $(B)$, and overall costs $(\mathbf{C})$ at 3 months after discharge following surgery for DS, among patients in the NDND, NDD, DND, and DD groups. 

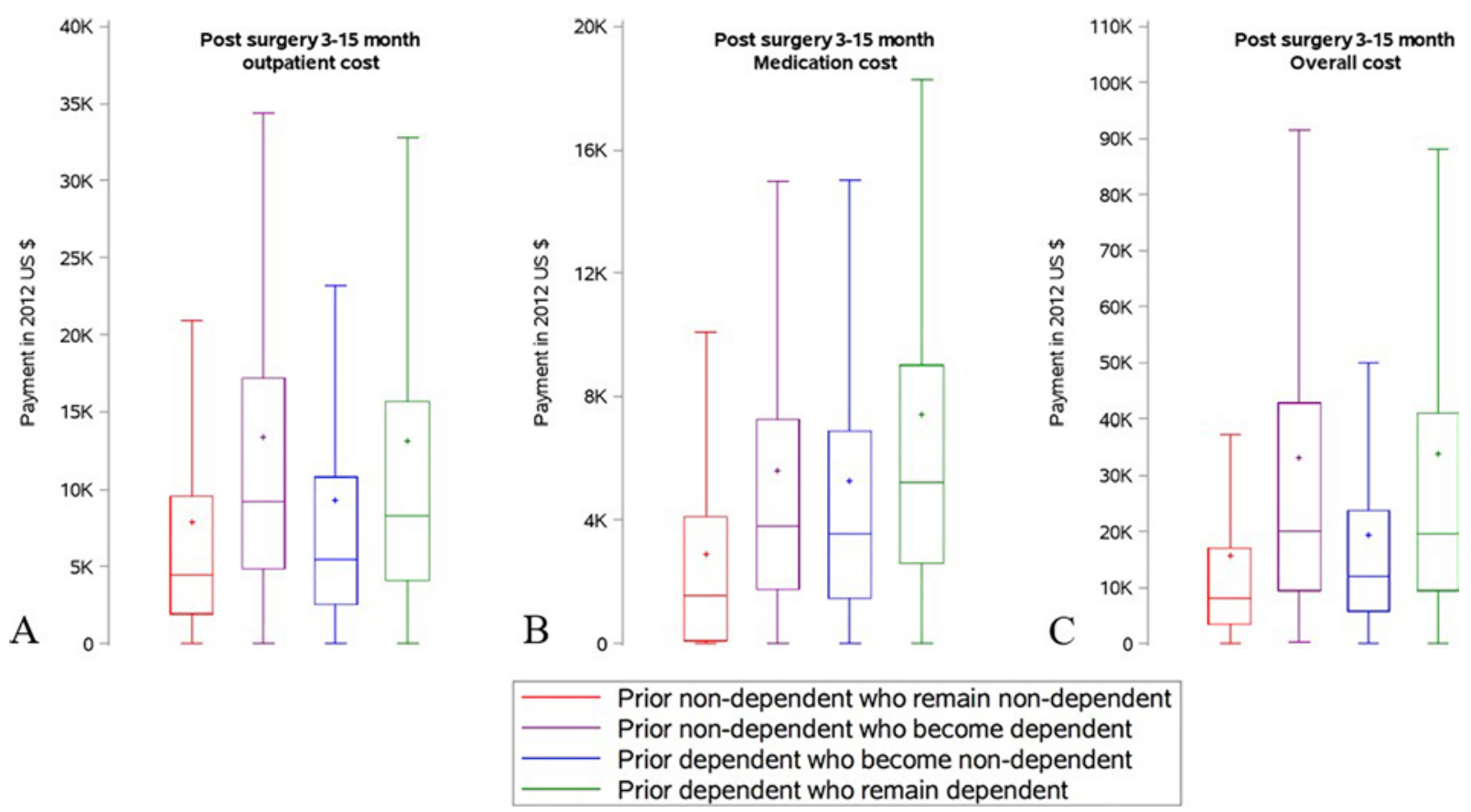

FIG. 3. Bar graph showing the median outpatient costs (A), medication costs $(\mathbf{B})$, and overall costs $(\mathbf{C})$ at 3-15 months after discharge following surgery for DS, among patients in the NDND, NDD, DND, and DD groups.

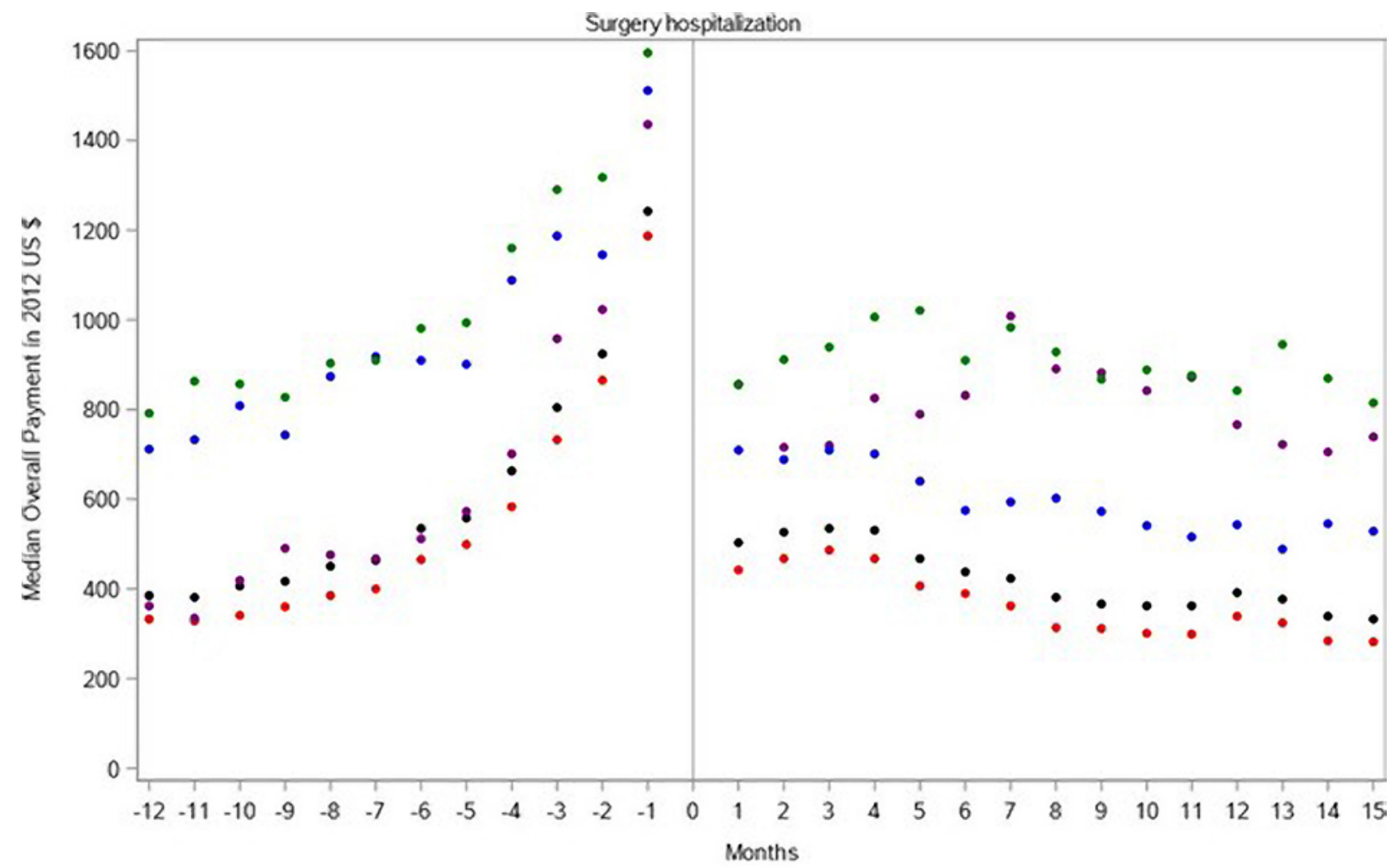

FIG. 4. Scatter diagram showing the preoperative and postoperative median overall payments among patients in the NDND, NDD, DND, and DD groups and the effect of surgery on the overall costs. 
of cervical and lumbar pathologies. However, this study did not examine postoperative opioid dependence and also analyzed heterogeneous spine pathologies. Based on our results, patients who were opioid dependent either prior to or after surgery for DS tend to have longer LOS following surgery for DS, compared with those who were opioid nondependent prior to and after surgery (NDND group). The overall incidence of in-hospital complications in our study was $9.5 \%$ compared to $22.5 \%$ in the study using the NIS database $(\mathrm{n}=48,911){ }^{23}$ This difference may be attributed to the selection bias in our study (patients with at least 12 months of follow-up prior to and after surgery were included). There was no difference in both in-hospital and 30-day complication rates among different groups in our study, which implies that opioid dependence has no correlation with complications following surgery for DS.

In our study, the overall median cost of index hospitalization was $\$ 40,095$. Patients in the DD group had a median cost of $\$ 43,524$, compared with $\$ 39,654$ for patients in group NDND, $\$ 41,052$ in group NDD, and \$41,027 in group DND $(p=0.0351)$. However, there was no significant difference among the groups on adjusted analysis ( $p$ $>0.05$ ). Another NIS-based study reported a weighted index hospitalization cost of $\$ 61,766$ in patients undergoing surgery for DS. ${ }^{23}$ Another study reported that combined medical and pharmaceutical expenditure during the 2-year period following lumbar fusion surgeries for degenerative conditions was $\$ 12,283 /$ individual. ${ }^{21}$ Similarly, we found that additional combined median inpatient, outpatient, and medication costs during the 15 -month period after the index hospitalization cost of the surgery for DS was $\$ 11,255$ per patient.

Also in our study, patients in the DD group had 2.1 and 2.8 times higher overall costs at 3 months and 3-15 months, respectively, compared to patients in the NDND group $(\mathrm{p}<0.001)$. This finding has important implications for routine clinical practice; various measures to reduce opioid dependence (psychiatric consultation, behavior therapy, etc.) should be considered prior to elective spine surgery. These measures can help reduce overall health care costs in this patient population.

\section{Limitations of the Study}

A major advantage of using the MarketScan database is that it provides longitudinal information related to inpatient and outpatient services that are inclusive of medication refills and health care utilization costs, in a large sample size across the US, thereby providing an opportunity to answer a clinically relevant question on a wider scale.

The limitations of using such a database include the retrospective nature of the study and inherent limitations associated with such methodology. Moreover, this database is based on insurance claims, which were used to charge in-hospital/outpatient services and medication costs during the treatment. Therefore, this study did not take into account the clinical scenario and thought process in the management as well the clinical follow-up of an individual patient. Other potential limitations include coding errors, inability to track the "actual consumption" of the opioid by the patient, and inability to capture the patients who were obtaining illegal opioids, which may over- or underestimate the actual clinical scenario. Despite these limitations, our study provides an in-depth analysis of the impact of opioid dependency on health care utilization at different time points in patients undergoing surgery for DS.

\section{Conclusions}

Patients who continued to be opioid dependent or become opioid dependent following surgery for DS incur significant health care utilization and costs at 3 months and 3-15 months. These findings can be used to formulate strategies to reduce the health care costs in this patient population, given the current scenario of the opioid crisis.

\section{References}

1. Albert TJ, Pinto M, Denis F: Management of symptomatic lumbar pseudarthrosis with anteroposterior fusion. A functional and radiographic outcome study. Spine (Phila Pa 1976) 25:123-130, 2000

2. Ambekar S, Sharma M, Kukreja S, Nanda A: Complications and outcomes of surgery for spinal meningioma: a Nationwide Inpatient Sample analysis from 2003 to 2010. Clin Neurol Neurosurg 118:65-68, 2014

3. Armaghani SJ, Even JL, Zern EK, Braly BA, Kang JD, Devin CJ: The evaluation of donor site pain after harvest of tricortical anterior iliac crest bone graft for spinal surgery: a prospective study. Spine (Phila Pa 1976) 41:E191-E196, 2016

4. Armaghani SJ, Lee DS, Bible JE, Archer KR, Shau DN, Kay $\mathrm{H}$, et al: Preoperative opioid use and its association with perioperative opioid demand and postoperative opioid independence in patients undergoing spine surgery. Spine (Phila Pa 1976) 39:E1524-E1530, 2014

5. Armaghani SJ, Lee DS, Bible JE, Shau DN, Kay H, Zhang $\mathrm{C}$, et al: Increased preoperative narcotic use and its association with postoperative complications and length of hospital stay in patients undergoing spine surgery. Clin Spine Surg 29:E93-E98, 2016

6. Cauley CE, Anderson G, Haynes AB, Menendez M, Bateman BT, Ladha K: Predictors of in-hospital postoperative opioid overdose after major elective operations: a nationally representative cohort study. Ann Surg 265:702-708, 2017

7. Cepeda MS, Fife D, Ma Q, Ryan PB: Comparison of the risks of opioid abuse or dependence between tapentadol and oxycodone: results from a cohort study. J Pain 14:1227-1241, 2013

8. Connolly J III, Javed Z, Raji MA, Chan W, Kuo YF, Baillargeon J: Predictors of long-term opioid use following lumbar fusion surgery. Spine (Phila Pa 1976) 42:1405-1411, 2017

9. Deyo RA, Gray DT, Kreuter W, Mirza S, Martin BI: United States trends in lumbar fusion surgery for degenerative conditions. Spine (Phila Pa 1976) 30:1441-1447, 2005

10. Elixhauser A, Steiner C, Harris DR, Coffey RM: Comorbidity measures for use with administrative data. Med Care 36:8-27, 1998

11. Finucane TE, Christmas C, Travis K: Tube feeding in patients with advanced dementia: a review of the evidence. JAMA 282:1365-1370, 1999

12. Florence CS, Zhou C, Luo F, Xu L: The economic burden of prescription opioid overdose, abuse, and dependence in the United States, 2013. Med Care 54:901-906, 2016

13. Hansen LG, Chang S: Health Research Data for the Real World: The Marketscan Databases. Ann Arbor: Truven Health Analytics, 2012

14. Helmerhorst GT, Vranceanu AM, Vrahas M, Smith M, Ring D: Risk factors for continued opioid use one to two months 
after surgery for musculoskeletal trauma. J Bone Joint Surg Am 96:495-499, 2014

15. Jacobsen S, Sonne-Holm S, Rovsing H, Monrad H, Gebuhr $\mathrm{P}$ : Degenerative lumbar spondylolisthesis: an epidemiological perspective: the Copenhagen Osteoarthritis Study. Spine (Phila Pa 1976) 32:120-125, 2007

16. Jiang X, Orton M, Feng R, Hossain E, Malhotra NR, Zager EL, et al: Chronic opioid usage in surgical patients in a large academic center. Ann Surg 265:722-727, 2017

17. Kepler CK, Vaccaro AR, Hilibrand AS, Anderson DG, Rihn JA, Albert TJ, et al: National trends in the use of fusion techniques to treat degenerative spondylolisthesis. Spine (Phila Pa 1976) 39:1584-1589, 2014

18. Littell RC, Stroup WW, Freund RJ: SAS for Linear Models, ed 4. Cary, NC: SAS Institute, 2002

19. Logan J, Liu Y, Paulozzi L, Zhang K, Jones C: Opioid prescribing in emergency departments: the prevalence of potentially inappropriate prescribing and misuse. Med Care 51:646-653, 2013

20. Martin BC, Fan MY, Edlund MJ, Devries A, Braden JB, Sullivan MD: Long-term chronic opioid therapy discontinuation rates from the TROUP study. J Gen Intern Med 26:14501457,2011

21. Mino DE, Munterich JE, Castel LD: Lumbar fusion surgery for degenerative conditions is associated with significant resource and narcotic use 2 years postoperatively in the commercially insured: a medical and pharmacy claims study. J Spine Surg 3:141-148, 2017

22. Nguyen TH, Randolph DC, Talmage J, Succop P, Travis R: Long-term outcomes of lumbar fusion among workers' compensation subjects: a historical cohort study. Spine (Phila Pa 1976) 36:320-331, 2011

23. Norton RP, Bianco K, Klifto C, Errico TJ, Bendo JA: Degenerative spondylolisthesis: an analysis of the Nationwide Inpatient Sample Database. Spine (Phila Pa 1976) 40:1219-1227, 2015

24. Oderda GM, Lake J, Rüdell K, Roland CL, Masters ET: Economic burden of prescription opioid misuse and abuse: a systematic review. J Pain Palliat Care Pharmacother 29:388-400, 2015

25. Parikh AA, Robinson J, Zaydfudim VM, Penson D, Whiteside MA: The effect of health insurance status on the treatment and outcomes of patients with colorectal cancer. J Surg Oncol 110:227-232, 2014

26. Paulozzi L, Baldwin G, Franklin G, Kerlikowske RG, Jones CM, Ghiya N, et al: CDC Grand Rounds: Prescription drug overdoses - a U.S. epidemic. MMWR Morb Mortal Wkly Rep 61:10-13, 2012

27. Quan H, Sundararajan V, Halfon P, Fong A, Burnand B, Luthi JC, et al: Coding algorithms for defining comorbidities in ICD-9-CM and ICD-10 administrative data. Med Care 43:1130-1139, 2005

28. Schoenfeld AJ, Nwosu K, Jiang W, Yau AL, Chaudhary MA, Scully RE, et al: Risk factors for prolonged opioid use following spine surgery, and the association with surgical intensity, among opioid-naive patients. J Bone Joint Surg Am 99:1247-1252, 2017

29. Sharma M, Sonig A, Ambekar S, Nanda A: Discharge dispositions, complications, and costs of hospitalization in spinal cord tumor surgery: analysis of data from the United States Nationwide Inpatient Sample, 2003-2010. J Neurosurg Spine 20:125-141, 2014

30. Sharma M, Ugiliweneza B, Aljuboori Z, Nuno MA, Drazin $\mathrm{D}$, Boakye M: Factors predicting opioid dependence in patients undergoing surgery for degenerative spondylolisthesis: analysis from Market Scan Database. J Neurosurg Spine [in press], 2018

31. Soneji N, Clarke HA, Ko DT, Wijeysundera DN: Risks of developing persistent opioid use after major surgery. JAMA Surg 151:1083-1084, 2016

32. Stokes ME, Davis CS, Koch GG: Categorical Data Analysis Using the SAS System, ed 2. Cary, NC: SAS Institute, 2000

33. Walid MS, Hyer L, Ajjan M, Barth AC, Robinson JS Jr: Prevalence of opioid dependence in spine surgery patients and correlation with length of stay. J Opioid Manag 3:127-128, 130-122, 2007

\section{Disclosures}

The authors report no conflict of interest concerning the materials or methods used in this study or the findings specified in this paper.

\section{Author Contributions}

Conception and design: Sharma. Analysis and interpretation of data: Sharma. Drafting the article: Sharma, Aljuboori. Critically revising the article: Boakye, Sharma, Ugiliweneza. Reviewed submitted version of manuscript: all authors. Statistical analysis: Ugiliweneza. Administrative/technical/material support: Boakye. Study supervision: Boakye.

\section{Supplemental Information Videos}

Video Abstract. https://vimeo.com/261518165.

\section{Correspondence}

Maxwell Boakye: University of Louisville, School of Medicine, Louisville, KY. maxwell.boakye@ulp.org. 